\title{
Clinical instability of breast cancer markers is reflected in long-term in vitro estrogen deprivation studies
}

\author{
Jelena Milosevic ${ }^{1 \dagger}$, Johanna Klinge ${ }^{1 \dagger}$, Anna-Lena Borg ${ }^{1}$, Theodoros Foukakis ${ }^{1,2}$, Jonas Bergh ${ }^{1,2,3}$ \\ and Nicholas P Tobin ${ }^{1 *}$
}

\begin{abstract}
Background: Long-term estrogen deprivation models are widely employed in an in vitro setting to recapitulate the hormonal milieu of breast cancer patients treated with endocrine therapy. Despite the wealth information we have garnered from these models thus far, a comprehensive time-course analysis of the estrogen (ER), progesterone (PR), and human epidermal growth factor 2 (HER-2/neu) receptors on the gene and protein level, coupled with expression array data is currently lacking. We aimed to address this knowledge gap in order to enhance our understanding of endocrine therapy resistance in breast cancer patients.
\end{abstract}

Methods: ER positive MCF7 and BT474 breast cancer cells were grown in estrogen depleted medium for 10 months with the ER negative MDA-MB-231 cell line employed as control. ER, PR and HER-2/neu expression were analysed at defined short and long-term time points by immunocytochemistry (ICC), and quantitative real-time RTPCR (qRT-PCR). Microarray analysis was performed on representative samples.

Results: MCF7 cells cultured in estrogen depleted medium displayed decreasing expression of ER up to 8 weeks, which was then re-expressed at 10 months. PR was also down-regulated at early time points and remained so for the duration of the study. BT474 cells generally displayed no changes in ER during the first 8 weeks of deprivation, however its expression was significantly decreased at 10 months. PR expression was also down-regulated early in BT474 samples and was absent at later time points. Finally, microarray data revealed that genes and cell processes down-regulated in both cell lines at 6 weeks overlapped with those down-regulated in aromatase inhibitor treated breast cancer patients.

Conclusions: Our data demonstrate that expression of ER, PR, and cell metabolic/proliferative processes are unstable in response to long-term estrogen deprivation in breast cancer cell lines. These results mirror recent clinical findings and again emphasize the utility of LTED models in translational research.

\section{Background}

The pathogenesis of breast cancer is a complex, multistep process involving multiple genetic changes. A major risk factor associated with the development of the disease is the duration of exposure to estrogens, the length of which is increased in women experiencing early menarche and/ or late menopause. Estrogens are steroid hormones that play important roles in the growth and development of

\footnotetext{
*Correspondence: tobin.nick@gmail.com

${ }^{\dagger}$ Equal contributors

${ }^{1}$ Cancer Center Karolinska, Karolinska Institutet and University Hospital, Stockholm S-171 76, Sweden

Full list of author information is available at the end of the article
}

the mammary gland and it is well established that the growth of breast cancer cell lines in culture or in ovariectomized nude mice is stimulated by estrogens [1-3].

Approximately two-thirds of all breast cancer tumours are ER-positive [4-6] and more than 50\% of these are also PR-positive [7]. Both receptors are useful in predicting response to endocrine therapy [5,7-9] and in general ER-negative tumours are associated with early recurrence and poor patient survival relative to those that are ER-positive $[5,8,9]$. Despite clinical advances of ERtargeted therapy, de novo and acquired resistance to all forms of endocrine therapy remains a great obstacle $[8,9]$. Complicating matters, we and others have shown in

\section{Biomed Central}


mostly retrospective studies, that expression of ER and PR are unstable during tumour progression from a primary lesion to its corresponding metastasis [10-13].

Long-term estrogen deprived (LTED) cell lines can serve as an in vitro model mimicking the hormonal milieu of breast cancer cells in oophorectomized premenopausal women, postmenopausal women and/or patients treated with primary endocrine therapy, in particular aromatase inhibitors (AIs) [14]. Of note, the use of AIs in place of traditional endocrine treatments results in a statistically significant survival gain (HR 0.90, 95\% CI 0.84 to 0.97 ) [15].

Whilst previous studies have examined ER, PR and HER-2/neu expression in an LTED setting, no comprehensive gene and protein analysis has been performed on all three markers. As such, our descriptive study addresses this knowledge gap by determining the levels of ER, PR and HER-2/neu gene and protein expression in two ER-positive and one ER-negative cell line at multiple time points, coupled with gene expression array profiling, all in a well-described LTED model [16-20]. Adding further clinical relevance to our analysis, we related our expression array findings to publicly available array data of breast cancer patients treated with an aromatase inhibitor. Our work highlights the unstable nature of ER and PR expression under conditions of estrogen deprivation, and demonstrates the significant overlap of genes altered in LTED cell lines and AI-treated patients.

\section{Methods}

\section{Cell culture}

A long-term estrogen deprivation (LTED) model was used to study the three commonly used breast cancer cell lines MCF7, BT474 and MDA-MB-231 [7,8]. MCF7 and MDA-MB-231 cells were newly purchased from Sigma-Aldrich and BT474 cells from the American Type Culture Collection (ATCC). Control and LTED cells were routinely maintained in phenol red containing MEM or DMEM supplemented with 10\% fetal bovine serum (FBS) or phenol red-free MEM or DMEM supplemented with $10 \%$ dextran-coated charcoal-stripped FBS (DCC-FBS) to remove substantial amounts of estrogen, respectively. Each culture medium was further supplemented with $100 \mathrm{IE} / \mathrm{ml}$ penicillin and $100 \mu \mathrm{l} / \mathrm{ml}$ streptomycin. All cells were grown at $37^{\circ} \mathrm{C}$ in a humidified atmosphere of $5 \% \mathrm{CO}_{2}$ and $95 \%$ air.

\section{Immunocytochemistry}

50000 cells per cell line (MCF7, BT474 and MDA-MB-231 cells) were attached to slides (ChemMateTM Capillary Gap Microscope Slides, DAKO) by centrifuging them in a Cytospin 3 centrifuge (Shandon, Thermo Electron corporation, Waltham, Massachusetts), at $1000 \mathrm{rpm}$ for 4 minutes in room temperature. The slides were then fixed in 4\% formalin for 10 minutes at room temperature, followed by PBS for 10 minutes, methanol for 4 minutes in $-20^{\circ} \mathrm{C}$, and acetone for 1 minute in $-20^{\circ} \mathrm{C}$, before being placed in TBS. Automatic immunostaining was performed in a DAKO Tech Mate instrument (DAKO, Glostrup, Denmark). Staining of ER and PR was done using the recommended DAKO ChemMate Detection Kit (Peroxidase/DAB Rabbit/Mouse). The MDA-MB-231 cell line served as negative control for ER, PR and HER-2/neu expression. MCF7 cell line was used as positive control for ER expression, while BT474 cell line served as positive control for PR and HER-2/neu expression.

Immunoslides were assessed in a microscope by counting of positive cells and degree of staining. We used a modified $\mathrm{H}$ score system, using the formula: $\mathrm{H}$ score $=(0 \times \%$ tumour cells negative $)+(1.5 \times \%$ tumour cells moderately positive $)+(3 \times \%$ tumour cells strongly positive), giving a range $0-300$. Five hundred cells were counted per slide. Two observers (JM and JK) evaluated the immunoslides, and the final score was calculated by taking the mean score. If the ratio between two scores was higher than 1.5 , the slides were re-evaluated to reach consensus.

The following primary antibodies were used for immunocytochemical analyses: Monoclonal mouse antihuman progesterone receptor (PR) antibody (Clone PgR 636, DAKO, Glostrup, Denmark), diluted 1:1000, monoclonal mouse anti-human estrogen receptor antibody NCL-ER-6 F11 (NovoCastra Laboratories Ltd, Newcastle, UK), diluted 1:50, monoclonal mouse anti-human HER-2 (c-erbB-2 Oncoprotein) antibody (NCL-CB11), diluted 1:250 (Novocastra Laboratories Ltd., Newcastle, UK).

\section{RNA isolation}

RNA extraction was performed according to the RNeasy mini protocol (Qiagen, Germany). Briefly, one to five millions cells were collected for isolation of RNA from each sample before being applied to the MicroSpin affinity columns in the Qiagen kit. The quality of RNA was assessed using an Agilent 2100 bioanalyzer (Agilent Technologies, Rockville, MD, USA).

\section{Quantitative real-time $\mathrm{PCR}$ analysis}

The mRNA expression levels of ESR1, PGR, ERBB2 and an endogenous housekeeping gene encoding for $18 \mathrm{~S}$ ribosomal RNA as a reference were quantified using TaqMan $^{\bullet}$ technology on an ABI PRISM 7500 sequence detection systems (PE Applied Biosystems). Sequencespecific primers and probes were selected from the Assayon-Demand products (Applied Biosystems), including ESR1 (assay ID: Hs01046817_m1), PGR (Hs00172183_m1), ERBB2 (Hs00170433_m1) and 18S ribosomal RNA (Hs9 9999901_s1). All qRT-PCR experiments included a no template control and were performed at least in duplicate. 


\section{Microarray analysis}

A total of eight samples, four from each cell line (control, 2 days, 6 weeks and 10 months), were selected for microarray analysis, performed at the core facility for Bioinformatics and Expression Analysis (BEA) at Karolinska Institutet. Briefly, biotinylated cRNA was hybridized to HG-U133 Plus 2.0 oligonucleotide arrays (Affymetrix Incorporated, Santa Clara, CA, USA), washed and scanned according to the protocol recommended by the supplier. Gene Chip Operating Software (GCOS) was used for calculation of detection calls, signal values and for calculation of the target intensity scaling of each array to an identical value and quantification of the signal log ratio. An average signal log ratio value was calculated for all transcripts in the long-term estrogen deprived cell lines compared to the cell lines cultured in medium containing estrogen. A minimum signal log ratio of 0.7 in each of four pair-wise comparisons was set as a threshold for significant differential expression. The quality of the data was verified by correlation analysis and multidimensional scaling plots in $R$ statistical environment using Bioconductor packages (http://www.bioconductor.org). This data has been made publically available at NCBI GEO with series accession number GSE50820. Gene Ontology (GO) terms enriched in the lists of up-regulated and down-regulated genes including the 300 genes with highest SLR, were identified by Fisher's exact test. For comparison of genes significantly changed in response to estrogen silencing to those significantly altered in our LTED model, we accessed publically available data (Gene expression omnibus number: GSE27473) from the NCBI GEO repository. The data is taken from a publication by $\mathrm{Al}$ Saleh et al. [21] where gene expression changes are determined in MCF7 cells after estrogen receptor silencing. In order to directly compare with our data, we downloaded and re-analysed the dataset using the statistical parameters outlined above to determine genes significantly changed in response to estrogen silencing.

\section{Statistical analysis}

All statistical analysis were performed using SPSS data analysis statistics software system version 17.0 (SPSS Inc., Chicago, IL, USA), the statistics tool in Microsoft Excel or R. ANOVA with post-hoc Tukey was performed on $\mathrm{H}$-score and qPCR data and significance was calculated relative to day 0 control. Experimental results are expressed as mean \pm SEM, where applicable. $P$-values of $<0.05$ were considered statistically significant.

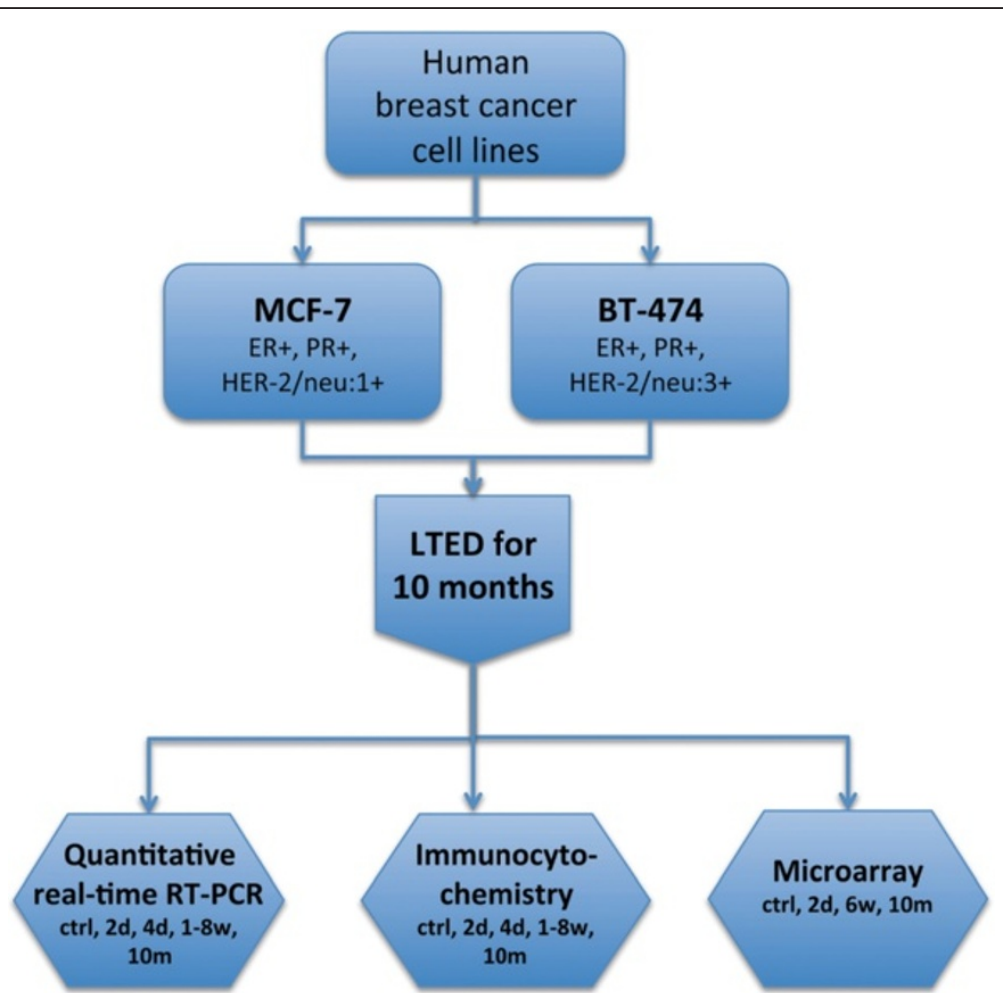

Figure 1 Experimental flowchart. ER, PR and HER-2/neu expression were analysed by qRT-PCR and immunocytochemistry (ICC) at selected time points (control, 2 days, 4 days, 1-8 weeks and 10 months after estrogen deprivation). Microarray analysis was performed on control cells and at 2 days, 6 weeks and 10 months after estrogen deprivation. 


\section{Results}

Re-expression of ER in an estrogen deprived environment occurs in the absence of PR in MCF7 cells

The breast cancer cell lines MCF7 and BT474 were cultured without estrogen for up to 10 months and examined by immunocytochemistry (quantified by $\mathrm{H}$-score) and qRT-PCR for changes in expression of ER, PR and HER-2/neu at the time points shown in our experimental overview (Figure 1). The ER, PR and HER-2/neu negative MDA-MB-231 cell line served as negative control. Cultured without estrogens, both ER positive cell lines initially stopped growing but MCF7 cells had returned to control levels of growth after ten months of continuous culture as determined by Ki67 (Additional file 1: Figure S1, upper panel), in line with previous studies [22]. BT474 cells displayed increased Ki67 expression after 10 months in LTED culture relative to 6 weeks, but had still not returned control levels of proliferation (Additional file 1: Figure S1, lower panel).

ER expression in MCF7 cells decreased gradually from 2 weeks to 8 weeks after estrogen deprivation, but was reexpressed at 10 months as determined by immunocytochemistry, qRT-PCR and $\mathrm{H}$-score (Figures 2A, 3A and Additional file 2: Figure S2A, respectively). Using identical methods, we found PR significantly down-regulated 2 days after estrogen deprivation (Figures 2B, 3B and Additional file 2: Figure S2B). After 1-2 weeks its expression was no longer detectable and remained so for the 10 month duration of the study. Changes in ER and PR protein expression at early time points were also confirmed by western blot (Additional file 3: Figure S3). Whilst we noted no change in HER-2/neu expression in response to estrogen deprivation by ICC (Additional file 4: Figure S4upper panel), we did find a small increase at the mRNA level (Figure 3C). It should be highlighted however, that given the scale of $E R B B 2$ expression it is unsurprising that this increase is not reflected by ICC. No expression of ER, PR or HER-2/neu was found in MDA-MB 231 cells as determined immunocytochemically (Additional file 5: Figure S5).

These results demonstrate the ability of MCF7 cells to up-regulate ER in the absence of PR under LTED conditions and moreover, highlight the unstable nature of ER and PR expression on both the gene and protein level when external estrogen levels are depleted.

\section{Reduced ER and PR expression in BT474 cells exposed to long-term estrogen deprived conditions}

In BT474 cells, no clear trend in ER expression was noted during the first 8 weeks of estrogen deprivation,

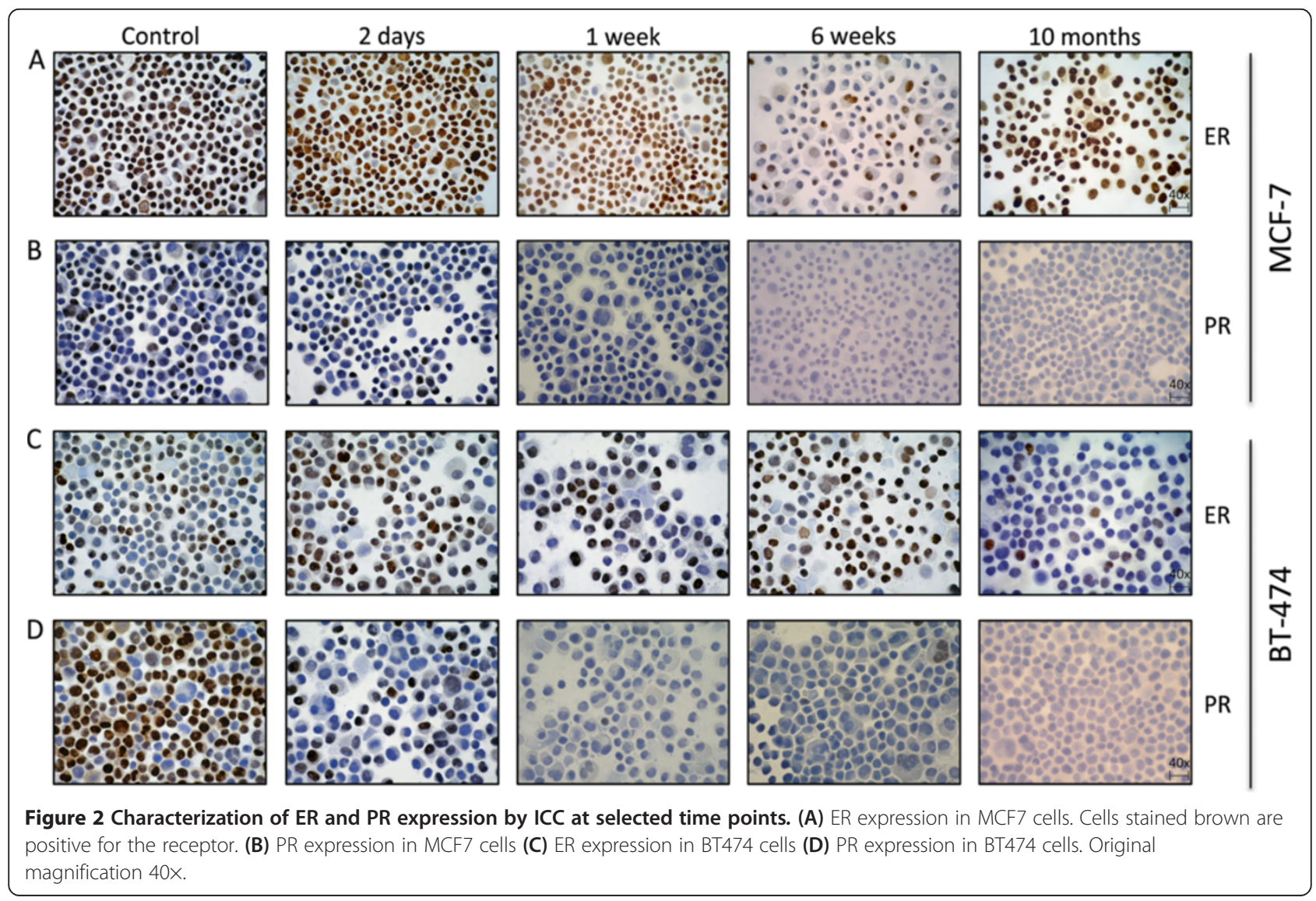




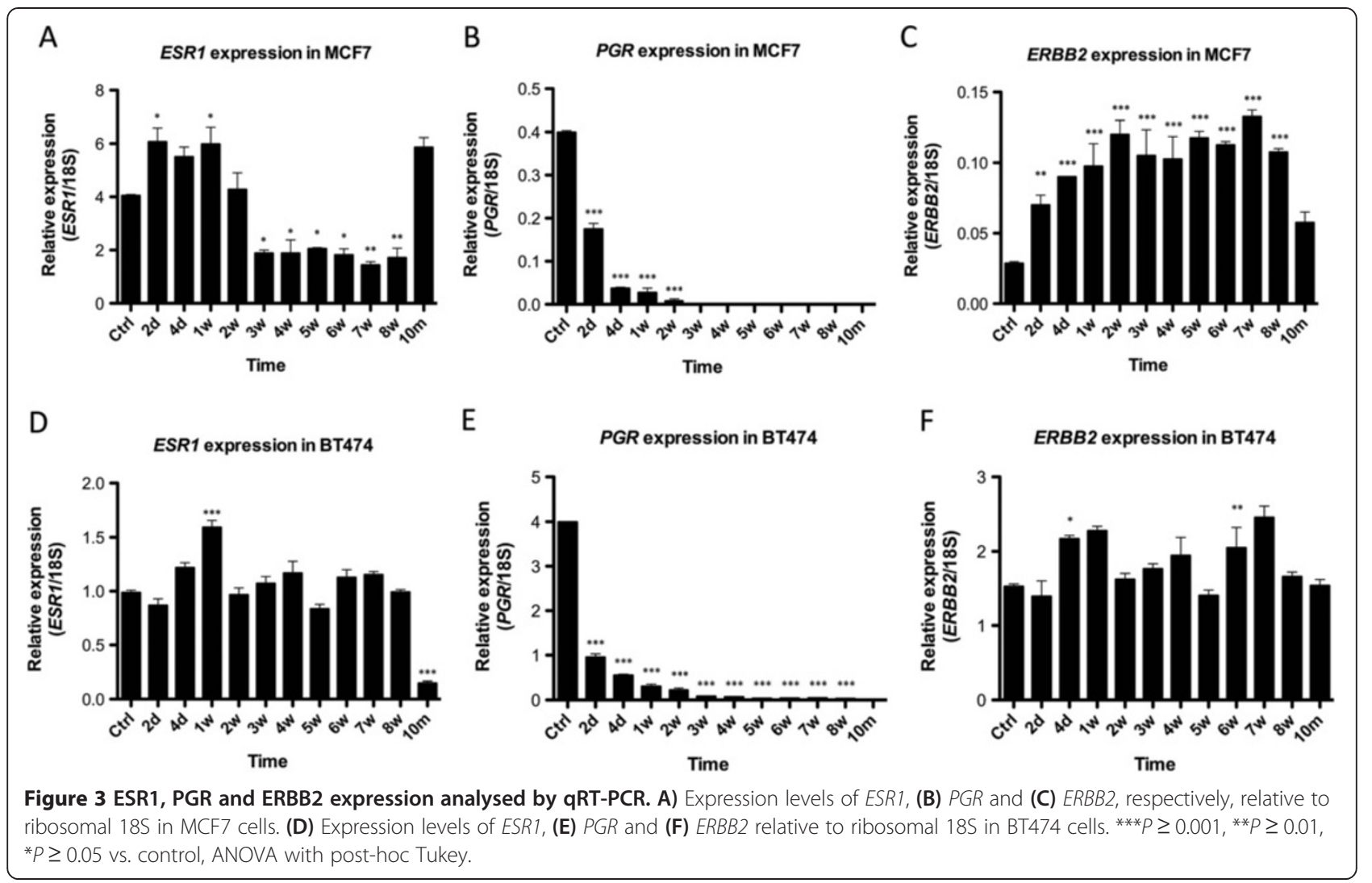

but a significant reduction was noted after 10 months by ICC and qRT-PCR (Figures 2C, 3D and Additional file 2: Figure S2C). Similarly to MCF7 cells, PR expression fell dramatically following 2 days of estrogen deprivation, was no longer expressed after 2-3 weeks, and remained undetectable on the protein level for the remainder of the study (Figures 2D, 3E and Additional file 2: Figure S2D). These changes were also confirmed by western blot at early time points (Additional file 3: Figure S3). HER-2/neu expression was not changed at any of the tested time points by ICC (Additional file 4: Figure S4, lower panels) but a weak trend towards increased ERBB2 was seen in the first 8 weeks of estrogen deprivation by qRT-PCR (Figure 3F).

These experiments again emphasize the instability of ER and PR in response to estrogen deprivation. Moreover, whilst the reduction in ER after 10 months in BT474 cells contrasts its increased expression at the same time-point in MCF7 cells, these results are consistent with the idea that individual cell lines can respond differently to LTED conditions [23].

Metabolic and cell cycle related genes down-regulated in initial response to estrogen deprivation are reupregulated in long-term culture Gene expression profiles were analysed in MCF7 and BT474 cells at 0 and 2 days, 6 weeks and 10 months after estrogen deprivation in order to examine gene expression changes in response to estrogen deprivation (see Figure 1, experimental overview).

In MCF7 cells, when comparing the 2 day and 6 week time points to control (0 days), the most down-regulated genes were those involved in metabolic processes and cell cycle, as expected. Figure 4A depicts the genes affected in cell cycle after 2 days and similar results were noted after 6 weeks (Additional file 6: Figure S6A). A full list of the cell cycle gene altered in MCF7 cells after 2 days LTED is provided in Additional file 7: Table S1. In the same samples the most notable up-regulated genes were TIMP2- which is involved in negative regulation of cell proliferation and NOTCH1. In the 10 month vs. control comparison we noted a reversal of these trends and genes involved metabolic and proliferative processes were up-regulated. Interestingly, genes down-regulated in the 10 month samples included those putatively involved in cell migration and motility (DNALI1, DNAH1, CXorf61), the apoptotic gene SULF1 and the PR gene PGR. Of note, a similar study of gene changes in MCF7 LTED cells over time has been previously performed, albeit in shorter time frame of 180 days [24]. Despite this difference, we saw a similar effect of LTED on the expression of the ESR1, MKI67, EGFR and RAF1 genes in our study as that found in the work of Aguilar et al. (Additional file 8: Figure S7), highlighting the reproducibility of LTED models. 


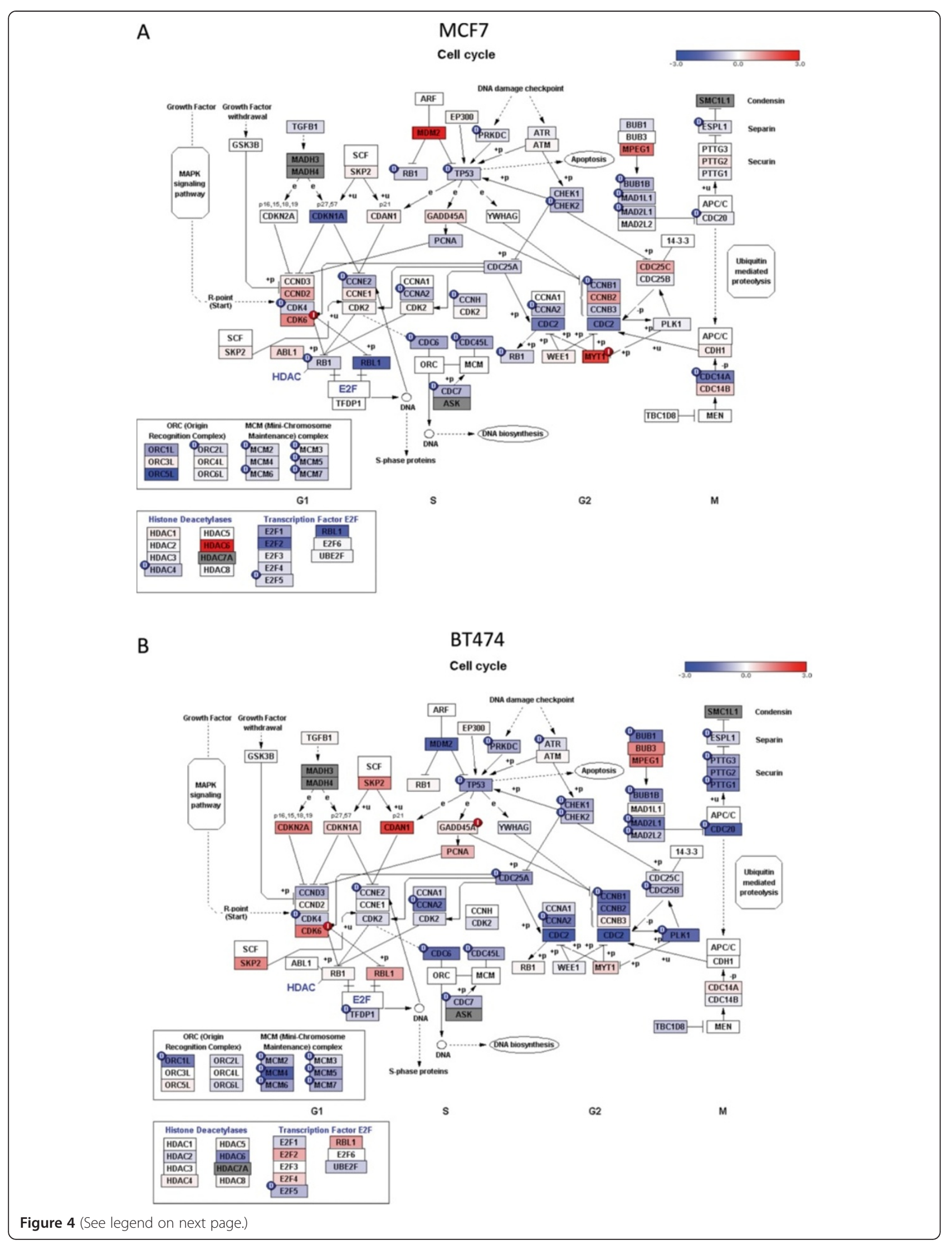


(See figure on previous page.)

Figure 4 Cell cycle genes affected in estrogen deprived cells. Red and blue boxes indicate up- and down regulated gene expression in response to estrogen deprivation relative to untreated control. Small circles (red and blue) mark a statistically significant increase or decrease in expression. (A) MCF7 cells at 2 days after estrogen deprivation versus control cells. (B) BT474 cells at 6 weeks after estrogen deprivation versus control cells.

In general, similar results were noted for BT474 cells at early time points, however the effect on cell cycle was less obvious after 2 days (Additional file 7: Figure S6B) but became apparent after 6 weeks (Figure 4B). A full list of the cell cycle gene altered in BT474 cells after 6 weeks LTED is provided in Additional file 9: Table S2. Again, the 10 month vs. control comparison showed upregulation of genes involved in metabolic and proliferative processes (CXCR4, INHBA), and down-regulation of those involved in cell motility (KIF6, VIM, TUBA1A), apoptosis (SEMA6A) and the PGR gene.

Together, these results point to an early down-regulation of genes involved in metabolic processes and cell cycle, as would be expected from estrogen deprivation. In long-term LTED culture, the situation is reversed and genes involved in the same processes are up-regulated whilst notably, genes implicated in cell motility and epithelial-to-mesenchymal transition (VIM) are down-regulated, in line with the "go or grow" hypothesis [25].

\section{Strong similarity between cell line genes altered in response to estrogen deprivation and those found in Al- treated breast cancer patients}

Next, with the aim of comparing the observed gene expression changes following estrogen deprivation in breast cancer cells to patients who received aromatase inhibitor (AI) treatment, we analysed a publicly available array data set consisting of 58 postmenopausal breast cancer patients with array profiles assessed before and after neoadjuvant treatment with letrozole (Gene expression omnibus number: GSE5462) [26].

In order to determine if similar processes were affected between our cell lines in response to estrogen deprivation and AI treated patients, we performed gene ontology analysis on our day 2 vs. control gene expression from MCF7 (Additional file 10: Table S3) and BT474 (Additional file 11: Table S4) cells. We found that the most changed processes in our cell line model including metabolic pathways, cell cycle, DNA replication, developmental processes and ion transport were also significantly changed in AI treated patients (see Miller et al. [26]).

Next, we examined the specific genes that were differentially expressed in our cell line model with those significantly changed upon letrozole treatment (Miller et al. [26]). We found that 14 of the 52 genes displaying decreased expression in AI-treated patients were also downregulated in MCF7 cells after 2 days. This number rose to
25 out of 52 when considering genes down-regulated in MCF7s 6 weeks after estrogen deprivation (Figure 5A).

Similarly in BT474 cells after 2 days, only 2/52 genes overlapped with those down-regulated in AI patients, but this increased to $31 / 52$ when comparing to the 6 week estrogen deprived (Figure 5A) samples. Of note, 19/52 gene probes down-regulated in both BT474 and MCF7 cells at 6 weeks after estrogen deprivation were also down-regulated in AI-treated patients (Figure 5A).

Up-regulated genes showed a smaller overlap with patient data; in MCF7 cells 4/36 and 8/36 gene probes upregulated after 2 days and 6 weeks estrogen deprivation respectively were also up-regulated in AI treated patients (Figure 5B). In BT474 cells these numbers fell to 2/36 and $7 / 36$ gene probes after 2 days and 6 weeks respectively (Figure 5B). Two genes were up-regulated in both MCF7 and BT474 cells at 6 weeks (TGFBR2 and CLU) were also upregulated in AI treated patients (Figure $5 \mathrm{~B}$ ).

Finally, in order to determine if gene changes caused specifically by loss of estrogen receptor are also present in the genes of LTED cells and AI -treated patients, we utilised publically available data (Gene expression omnibus number: GSE27473) of MCF7 cells treated with siRNA against the estrogen receptor [21]. Notably, we found an overlap of 4 genes significantly up-regulated and 11 genes significantly down-regulated in all three datasets (Additional file 12: Table S5). Of the up regulated genes, both SNAI2 and TGFBR2 are associated with promotion of epithelial-to-mesenchymal transition, whilst among the down-regulated genes were those responsible for the suppression of EMT including RACGAP1, TFF3 and IRS1. These results again implicate the induction of EMT through loss of estrogen receptor, in line with the work of others [21].

Taken together these data lend weight to the ability of this established model to provide relevant translational information and further support its use as a testing ground for elucidation of factors that mediate anti-estrogen treatment resistance.

\section{Discussion}

In spite of the substantial progress that has been achieved in recent years in the treatment of hormone receptor positive breast cancer, de novo and acquired resistance to endocrine therapy is still a major clinical problem $[8,9]$. In this descriptive study, we employed a LTED model to gain a greater understanding of how estrogen deprivation impacts clinically relevant prognostic markers and gene expression 


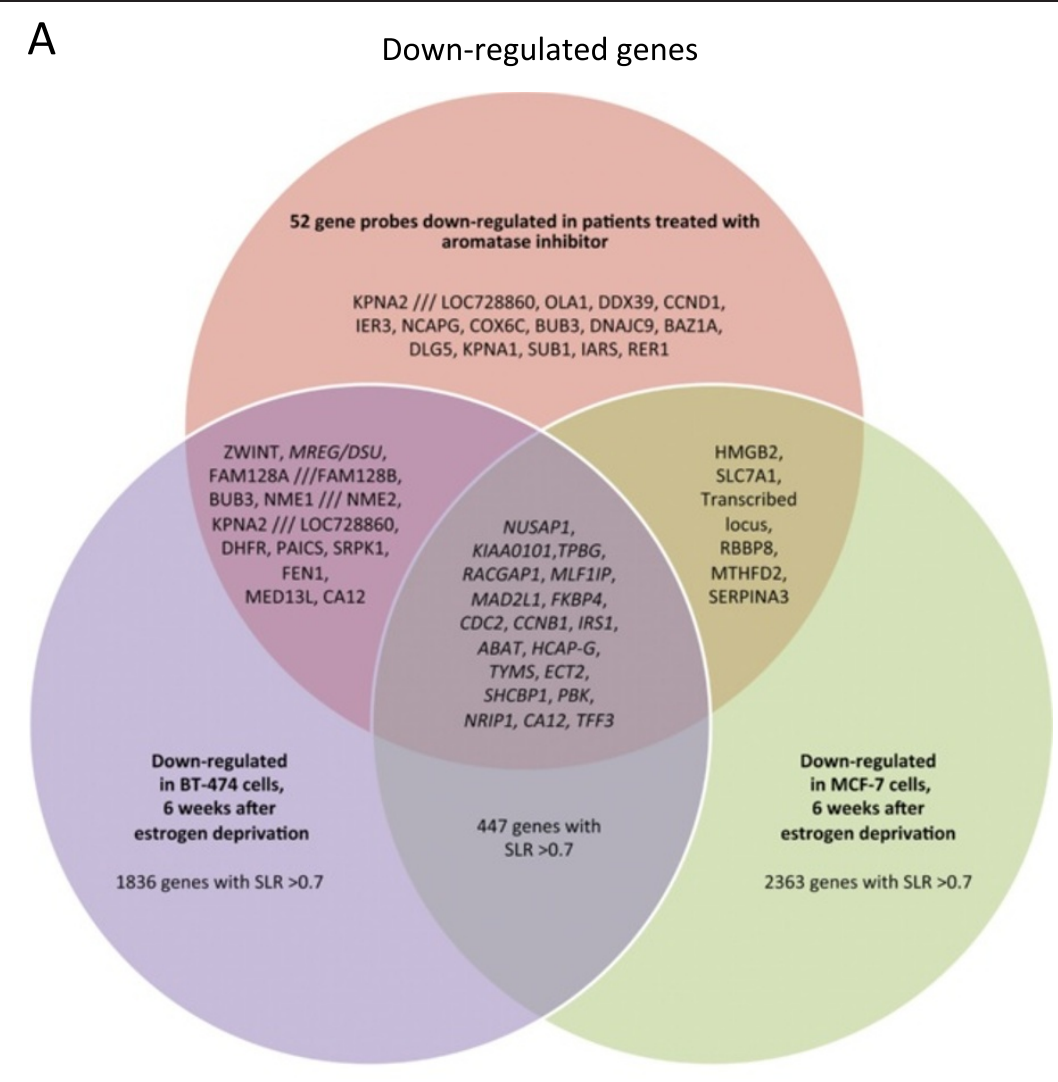

\section{B}

Up-regulated genes

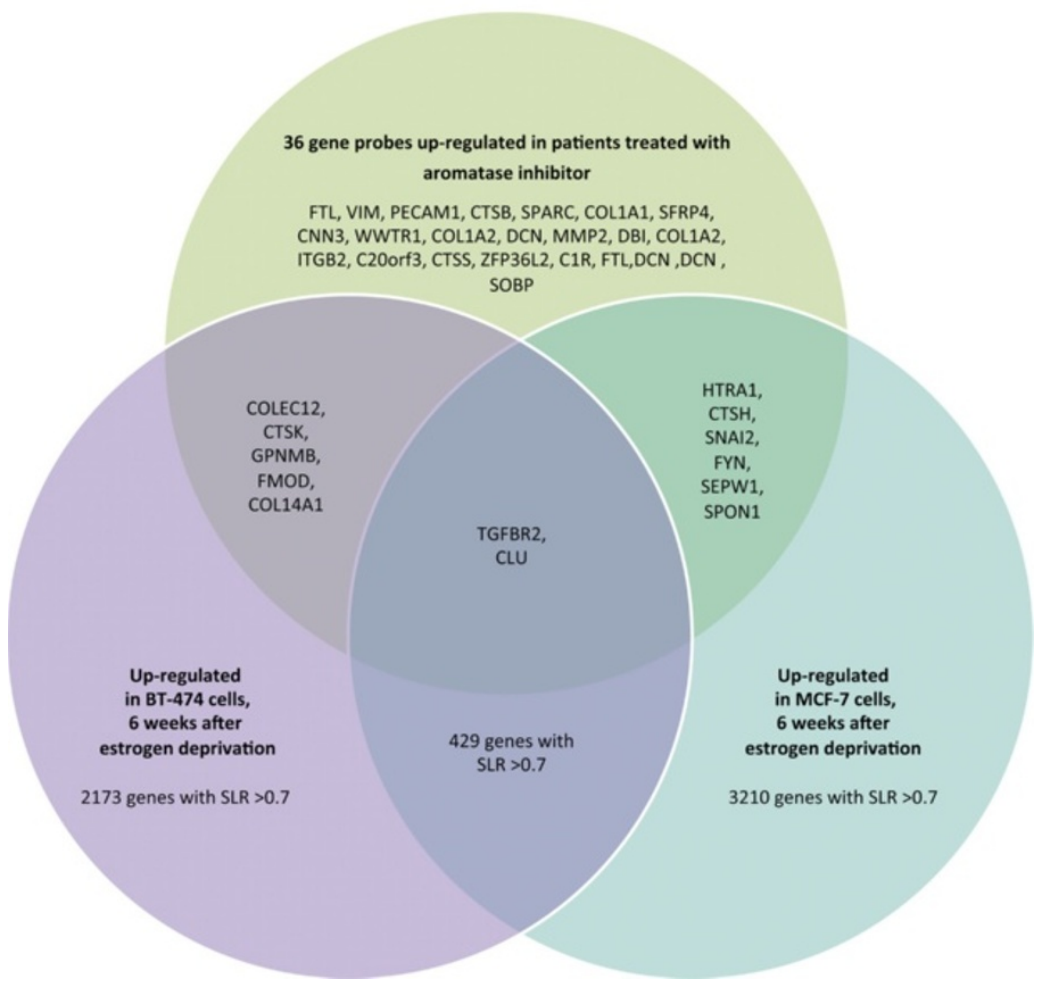

Figure $\mathbf{5}$ (See legend on next page.) 
(See figure on previous page.)

Figure 5 Venn diagram comparing significantly changed genes in MCF7, BT474 cell lines and Al-treated patients. (A) Genes significantly down-regulated in response to estrogen deprivation after 6 weeks vs. control in MCF7 and BT474 cells compared with those significantly downregulated in Al-treated patients (B) Genes significantly up-regulated in response to estrogen deprivation after 6 weeks vs. control in MCF7 and BT474 cells compared with those significantly up-regulated in Al-treated patients.

over time. To our knowledge, this is the first report to comprehensively investigate ER, PR and HER-2/neu expression along with qRT-PCR and gene expression array profiles at multiple early and late time points, in breast cancer cell lines after estrogen deprivation. Overall, our data are in line with previous reports showing that breast cancer cells can survive estrogen deprivation and re-grow, creating a phenotype that is likely less responsive to antihormonal therapy [27]. Additionally, due to the multiple consecutive time points examined, we note clear trends in how the expression of ER and PR change over time on both the gene and protein level. Lastly, we underline the similarities between the specific genes changed in our LTED cell lines and patients treated with aromatase inhibitors, demonstrating the strong translational value of this model, as others have also noted [23,24,28].

In order to put our work in the context of other studies and strengthen our findings, we compared our gene expression results to that of Aguilar et al., who performed a similar study in an MCF7 LTED model [24]. Through integrated aCGH and gene expression analysis the Aguilar study demonstrated that there may be shift towards a transcriptomic program in LTED cells that is independent of $\mathrm{ER} \alpha$ transcriptional function. Whilst we did not perform matching aCGH analysis on our LTED samples, and despite the differences in time points assessed in both studies, we did note similar changes in gene expression probes over time. Specifically, we noted analogous changes in the probes for ESR1, MKI67, EGFR and RAF1 (but not GATA3), thus lending support to hypotheses proposed by Aguilar et al.

Recent publications including two prospective studies, indicate lack of stability of ER and PR during tumour progression, in particular they seem to be altered when adjuvant therapies are given [29-31]. This loss of receptors, at least in the examined parts of the biopsies, may be a further factor involved in resistance to endocrine therapies. It is also apparent from these studies that ER and PR seem to be more discordant in patients receiving more abundant adjuvant therapies and a similar finding has been demonstrated with chemotherapy and trastuzumab in the comparison of HER-2/neu status in the primary tumour and the corresponding recurrence [31]. This clinical instability is reflected in our present cell line model, again underlining the suitability of LTED studies for investigating the time related alteration of receptors during conditions which mimic endocrine therapy with aromatase inhibition.

Previous studies have shown the propensity of breast cancer cells to adapt to conditions of long-term estrogen deprivation by up-regulating expression of ER, but not PR $[19,32]$, thus developing hypersensitivity to the mitogenic effect of estradiol. In our experiments, we observed a marked up-regulation of ER in the MCF7 but not BT474 cell line at 10 months after estrogen deprivation. Some reports claim that this estradiol hypersensitivity is not a consequence of ER-mediated gene transcription but rather related to activation of the MAPK/ERK [19] and EGFR/ ERBB/AKT pathways [24]. Similarly, recent evidence has also implicated a switch from ER $\alpha$ to $\mathrm{NOTCH}$ signalling in LTED cells [28], a finding supported by our analysis where we see an up-regulation of the NOTCH1 in MCF7 cells relative to control after 6 weeks of LTED culture.

The up-regulation of NOTCH1 fits well with our findings of increased expression of genes that promote EMT in both LTED MCF7 cells at 6 weeks and AI treated patients. Previous studies have linked induction of EMT under hypoxic conditions to Notch signalling [33], whilst ectopic expression of Notch1 intracellular domain (N1CD) has been demonstrated to trigger an EMT in epithelial cancer cells [34]. Of particular note, others have shown that a decrease in estrogen dependency is correlated with an increase of the EMT marker Snail1 in an MCF7 LTED model [35]. What these results mean in the context of AI treatment of breast cancer patients is difficult to ascertain. One might expect that as induction of EMT leads to an enhancement in the migratory capacity of cells, treating breast cancer patients with AIs would push tumour cells towards a more invasive metastatic phenotype. However, given the high success rates of endocrine treatments and reduced numbers of metastasis seen amongst these patients (relative to those who receive chemotherapy), this hypothesis would seem unlikely.

The down-regulation of PR following estrogen deprivation observed in our experiments could be caused by multiple cellular mechanisms. Cui et al. have shown that insulin-like growth factor-1 (IGF-1), independent of ER activity, considerably down-regulates PR through the PI3K (Akt/mTOR) pathway [36]. Along with others, they propose that low PR status may serve as an indicator of substantial activation of the growth factor signalling 
cascade, leading to hormonal therapy resistance [37-40]. However, our gene array data did not support any significant involvement of the PI3K/Akt pathway and as such the mechanisms governing loss of PR in our model will require further investigations.

\section{Conclusions}

Our data highlight the instability of ER, PR and metabolic/proliferative processes in response to short and long-term estrogen deprivation. Additionally we demonstrate considerable the overlap between genes altered in LTED culture and AI-treated breast cancer patients. These results further strengthen the use of LTED models as a valuable translational research tool to further our understanding of the major clinical obstacle that is hormonal resistance.

\section{Additional files}

Additional file 1: Figure S1. Characterization of Ki67 expression by ICC in MCF7 and BT474 cell lines at consecutive time points. Cells stained brown are positive for Ki67. Upper panel: MCF7 cells, Lower panel: BT474 cells. Original magnification 40x.

Additional file 2: Figure S2. Histograms of modified $\mathrm{H}$ score analysis. (A) Expression of ER and (B) PR in MCF7 cells. (C) Expression of ER and (D) PR in BT474 cells. ${ }^{* *} P \geq 0.001,{ }^{* *} P \geq 0.01,{ }^{*} P \geq 0.05$ vs. control, ANOVA with post-hoc Tukey.

Additional file 3: Figure S3. Western blots showing changes in ER and PR expression in response to estrogen deprivation in BT474 and MCF7 cells at early time points. $\beta$-actin is included as loading control and MDAMB-231 cells are included as negative control for ER and PR expression. Blots are representative.

Additional file 4: Figure S4. Characterization of HER-2/neu expression by ICC in MCF7 and BT474 cell lines at consecutive time points. Cells stained brown are positive for HER-2/neu receptor. Upper panel: MCF7 cells, Lower panel: BT474 cells. Original magnification 40x.

Additional file 5: Figure S5. Characterization of ER, PR and HER-2/neu expression by ICC in MDA-MB-231 cell line at consecutive time points. (A) Lack of ER expression (B) Lack of PR expression. (C) Lack of HER-2/neu expression. Original magnification 40X.

Additional file 6: Figure S6. Cell cycle genes affected in estrogen deprived cells. Red and blue boxes indicate up- and down regulated gene expression in response to estrogen deprivation. Small circles (red and blue) mark a statistically significant increase or decrease in expression. (A) MCF7 cells at 6 weeks after estrogen deprivation versus control cells (B) BT474 cells at 2 days after estrogen deprivation versus control cells.

Additional file 7: Table S1. Log fold change of cell cycle genes in MCF7 cells 2 days after estrogen deprivation versus control. This table displays all genes of the human KEGG annotated cell cycle pathway and their fold change after two days of estrogen deprivation relative to control, sorted according to $p$-value. Note, multiple affymetrix probes can map to the same gene. *Direction of change: $I=\operatorname{Increase}, \mathrm{D}=$ Decrease and $N C=$ No statistically significant change

Additional file 8: Figure S7. The effect of LTED on selected probesets. Here, in order to put our results in context with other scientific publications we reproduced the probeset plots of Aguilar et al. (A) ESR1 affymetrix probesets (B) MKI67 affymetrix probesets (C) Genes related to ER genomic function

Additional file 9: Table S2. Log fold change of cell cycle genes in BT474 cells 6 weeks after estrogen deprivation versus control. This table displays all genes of the human KEGG annotated cell cycle pathway and their fold change after 6 weeks of estrogen deprivation relative to control, sorted according to $\mathrm{p}$-value. Note, multiple affymetrix probes can map to the same gene. * Direction of change: $I=$ Increase, $D=$ Decrease and $N C=$ No statistically significant change.

Additional file 10: Table S3. MCF7 cells 2 days after estrogen deprivation versus control cells. The table represents the number of genes matching the 10 most commonly occurring $\mathrm{GO}$ terms in the $\mathrm{GO}$ molecular function, biological processes, and cellular component classes. The 300 genes with highest SLR were selected.

Additional file 11: Table S4. BT474 cells 2 days after estrogen deprivation versus control cells. The table represents the number of genes matching the 10 most commonly occurring $\mathrm{GO}$ terms in the GO molecular function, biological processes, and cellular component classes. The 300 genes with highest SLR were selected.

Additional file 12: Table S5. Genes in common amongst those significantly altered in all three analysed datasets: MCF7 LTED culture, ERsilenced MCF7 cells and breast cancer patients treated with aromatase inhibitors. We determined the genes most significantly altered in three datasets; our MCF7 LTED samples (control vs. 6 weeks, pvalue cutoff = 0.004), a publically available dataset of MCF7 cells where the ER has been silenced (control vs. silenced, pvalue cutoff $=0.004$, GSE27473) and a publically available dataset of breast cancer patients treated with aromatase inhibitors (GSE5462). We then determined the genes in common amongst those significantly altered in all three studies and present them here divided into those up and down regulated.

\section{Competing interests}

Professor Bergh receives research funding from Merck, paid to Karolinska Institutet and from Amgen, Roche, Sanofi-Aventis and Bayer, paid to Karolinska University Hospital. The authors have no other potential competing interest to disclose.

\section{Authors' contributions}

JM carried out the cell culturing, immunocytochemistry, $\mathrm{H}$ score analysis and qRT-PCR analysis, performed statistical analysis studies, participated in the interpretation of the data and drafted the manuscript. JK carried out $\mathrm{H}$ score analysis, participated in the interpretation of the data and helped to draft the manuscript. ALB helped with the immunocytochemistry analysis and isolation of RNA and DNA. TF participated in the GRT-PCR analysis. JB conceived of the study, participated in its design and coordination and revised the manuscript critically. NT participated in the interpretation of the data, design, performed the statistical analysis, and revised the manuscript critically. All authors read and approved the final manuscript.

\section{Acknowledgements}

This work was supported by grants from the Swedish Research Council, Gösta Miltons Donations fond, Karolinska Institutet Foundations, Swedish Cancer Society, Cancer Society in Stockholm, The King Gustaf V Jubilee Fund, Swedish Breast Cancer Association, Märit and Hans Rausing's Initiative Against Breast Cancer and BRECT.

\section{Author details}

'Cancer Center Karolinska, Karolinska Institutet and University Hospital, Stockholm S-171 76, Sweden. '2Department of Oncology and Pathology, Radiumhemmet, Karolinska Institutet and University Hospital, Stockholm Sweden. ${ }^{3}$ Honorary Professor, Manchester University, Manchester M20 4BX, England.

Received: 3 July 2013 Accepted: 8 October 2013

Published: 11 October 2013

\section{References}

1. Jordan VC: The ups and downs of the estrogen receptor. J Clin Oncol 2003, 21:3-4.

2. Song RX, Mor G, Naftolin F, McPherson RA, Song J, Zhang Z, Yue W, Wang J, Santen RJ: Effect of long-term estrogen deprivation on apoptotic responses of breast cancer cells to 17beta-estradiol. J Natl Cancer Inst 2001, 93:1714-1723. 
3. Lippert $\mathrm{C}$, Seeger $\mathrm{H}$, Mueck AO: The effect of endogenous estradiol metabolites on the proliferation of human breast cancer cells. Life Sci 2003, 72:877-883.

4. Osborne CK, Schiff R: Estrogen-receptor biology: continuing progress and therapeutic implications. J Clin Oncol 2005, 23:1616-1622.

5. Osborne CK: Steroid hormone receptors in breast cancer management. Breast Cancer Res Treat 1998, 51:227-238.

6. Kumar V, Green S, Stack G, Berry M, Jin JR, Chambon P: Functional domains of the human estrogen receptor. Cell 1987, 51:941-951.

7. Kim HJ, Cui X, Hilsenbeck SG, Lee AV: Progesterone receptor loss correlates with human epidermal growth factor receptor 2 overexpression in estrogen receptor-positive breast cancer. Clin Cancer Res 2006, 12(3 Pt 2):1013s-1018s.

8. Massarweh $\mathrm{S}$, Schiff R: Resistance to endocrine therapy in breast cancer: exploiting estrogen receptor/growth factor signaling crosstalk. Endocr Relat Cancer 2006, 13(Suppl 1):S15-S24.

9. Massarweh S, Schiff R: Unraveling the mechanisms of endocrine resistance in breast cancer: new therapeutic opportunities. Clin Cancer Res 2007, 13:1950-1954.

10. Konecny G, Pauletti G, Pegram M, Untch M, Dandekar S, Aguilar Z, Wilson C, Rong HM, Bauerfeind I, Felber M, Wang HJ, Beryt M, Seshadri R, Hepp H, Slamon DJ: Quantitative association between HER-2/neu and steroid hormone receptors in hormone receptor-positive primary breast cancer. J Natl Cancer Inst 2003, 95:142-153.

11. Tobin NP, Sims AH, Lundgren KL, Lehn S, Landberg G: Cyclin D1, Id1 and EMT in breast cancer. BMC Cancer 2011, 11:417.

12. Foukakis T, Astrom G, Lindstrom L, Hatschek T, Bergh J: When to order a biopsy to characterise a metastatic relapse in breast cancer. Ann Oncol 2012, 23(10):349-353.

13. Lindstrom LS, Karlsson E, Wilking UM, Johansson U, Hartman J, Lidbrink EK, Hatschek T, Skoog L, Bergh J: Clinically used breast cancer markers such as estrogen receptor, progesterone receptor, and human epidermal growth factor receptor 2 Are unstable throughout tumor progression. J Clin Oncol 2012, 30:2601-2608.

14. Dowsett M, Cuzick J, Ingle J, Coates A, Forbes J, Bliss J, Buyse M, Baum M, Buzdar A, Colleoni M, Coombes C, Snowdon C, Gnant M, Jakesz R, Kaufmann M, Boccardo F, Godwin J, Davies C, Peto R: Meta-analysis of breast cancer outcomes in adjuvant trials of aromatase inhibitors versus tamoxifen. J Clin Oncol 2010, 28:509-518.

15. Gibson L, Lawrence D, Dawson C, Bliss J: Aromatase inhibitors for treatment of advanced breast cancer in postmenopausal women. In Cochrane Database Syst Rev. John Wiley \& Sons, Ltd; 1996.

16. Song RX, Santen RJ, Kumar R, Adam L, Jeng MH, Masamura S, Yue W: Adaptive mechanisms induced by long-term estrogen deprivation in breast cancer cells. Mol Cell Endocrinol 2002, 193:29-42.

17. Martin LA, Farmer I, Johnston SR, Ali S, Marshall C, Dowsett M: Enhanced estrogen receptor (ER) alpha, ERBB2, and MAPK signal transduction pathways operate during the adaptation of MCF-7 cells to long term estrogen deprivation. J Biol Chem 2003, 278:30458-30468.

18. Yue W, Wang JP, Conaway MR, Li Y, Santen RJ: Adaptive hypersensitivity following long-term estrogen deprivation: involvement of multiple signal pathways. J Steroid Biochem Mol Biol 2003, 86:265-274.

19. Yue W, Wang JP, Conaway M, Masamura S, Li Y, Santen RJ: Activation of the MAPK pathway enhances sensitivity of MCF-7 breast cancer cells to the mitogenic effect of estradiol. Endocrinology 2002, 143:3221-3229.

20. Yue W, Fan P, Wang J, Li Y, Santen RJ: Mechanisms of acquired resistance to endocrine therapy in hormone-dependent breast cancer cells. J Steroid Biochem Mol Biol 2007, 106:102-110.

21. Al Saleh S, Al Mulla F, Luqmani YA: Estrogen receptor silencing induces epithelial to mesenchymal transition in human breast cancer cells. PLoS One 2011, 6:e20610

22. Chan CM, Martin L-A, Johnston SR, Ali S, Dowsett M: Molecular changes associated with the acquisition of oestrogen hypersensitivity in MCF-7 breast cancer cells on long-term oestrogen deprivation. J Steroid Biochem Mol Biol 2002, 81:333-341.

23. Miller TW, Hennessy BT, González-Angulo AM, Fox EM, Mills GB, Chen H, Higham C, García-Echeverría C, Shyr Y, Arteaga CL: Hyperactivation of phosphatidylinositol-3 kinase promotes escape from hormone dependence in estrogen receptor-positive human breast cancer. J Clin Investig 2010, 120:2406-2413.
24. Aguilar H, Solé X, Bonifaci N, Serra-Musach J, Islam A, López-Bigas N, Méndez-Pertuz M, Beijersbergen RL, Lázaro C, Urruticoechea A, Pujana MA: Biological reprogramming in acquired resistance to endocrine therapy of breast cancer. Oncogene 2010, 29:6071-6083.

25. Hatzikirou H, Basanta D, Simon M, Schaller K, Deutsch A: "Go or grow": the key to the emergence of invasion in tumour progression? Math Med Biol 2010, 29:49-65.

26. Miller WR, Larionov AA, Renshaw L, Anderson TJ, White S, Murray J, Murray E, Hampton G, Walker JR, Ho S, Krause A, Evans DB, Dixon JM: Changes in breast cancer transcriptional profiles after treatment with the aromatase inhibitor, letrozole. Pharmacogenet Genomics 2007, 17:813-826.

27. Jeng MH, Shupnik MA, Bender TP, Westin EH, Bandyopadhyay D, Kumar R, Masamura S, Santen RJ: Estrogen receptor expression and function in long-term estrogen-deprived human breast cancer cells. Endocrinology 1998, 139:4164-4174

28. Magnani L, Stoeck A, Zhang X, Lánczky A, Mirabella AC, Wang T-L, Gyorffy B, Lupien M: Genome-wide reprogramming of the chromatin landscape underlies endocrine therapy resistance in breast cancer. Proc Natl Acad Sci USA 2013, 110:E1490-E1499.

29. Amir E, Miller N, Geddie W, Freedman O, Kassam F, Simmons C, Oldfield M, Dranitsaris G, Tomlinson G, Laupacis A, Tannock IF, Clemons M: Prospective study evaluating the impact of tissue confirmation of metastatic disease in patients with breast cancer. J Clin Oncol 2011, 30:587-592.

30. Thompson AM, Jordan LB, Quinlan P, Anderson E, Skene A, Dewar JA, Purdie CA: Prospective comparison of switches in biomarker status between primary and recurrent breast cancer: the breast recurrence in tissues study (BRITS). Breast Cancer Res 2010, 12:R92.

31. Niikura N, Liu J, Hayashi N, Mittendorf EA, Gong Y, Palla SL, Tokuda Y, Gonzalez-Angulo AM, Hortobagyi GN, Ueno NT: Loss of human epiderma growth factor receptor 2 (HER2) expression in metastatic sites of HER2overexpressing primary breast tumors. J Clin Oncol 2011, 30:593-599.

32. Weigel MT, Ghazoui Z, Dunbier A, Pancholi S, Dowsett M, Martin LA: Preclinical and clinical studies of estrogen deprivation support the PDGF/Abl pathway as a novel therapeutic target for overcoming endocrine resistance in breast cancer. Breast Cancer Res 2012, 14:R78.

33. Chen J, Imanaka N, Chen J, Griffin JD: Hypoxia potentiates notch signaling in breast cancer leading to decreased E-cadherin expression and increased cell migration and invasion. Br J Cancer 2009, 102:351-360.

34. Bolos V, Mira E, Poveda BM, Luxan G, Canamero M, Martinez-A C, Manes S, de la Pompa JL: Notch activation stimulates migration of breast cancer cells and promotes tumor growth. Breast Cancer Res 2013, 15:R54.

35. Scherbakov AM, Andreeva OE, Shatskaya VA, Krasil'nikov MA: The relationships between snail1 and estrogen receptor signaling in breast cancer cells. J Cell Biochem 2012, 113:2147-2155.

36. Cui X, Schiff R, Arpino G, Osborne CK, Lee AV: Biology of progesterone receptor loss in breast cancer and its implications for endocrine therapy. J Clin Oncol 2005, 23:7721-7735.

37. Cui $X$, Zhang P, Deng W, Oesterreich S, Lu Y, Mills GB, Lee AV: Insulin-like growth factor-I inhibits progesterone receptor expression in breast cancer cells via the phosphatidylinositol 3-kinase/Akt/mammalian target of rapamycin pathway: progesterone receptor as a potential indicator of growth factor activity in breast cancer. Mol Endocrinol 2003, 17:575-588.

38. Dowsett M, Harper-Wynne C, Boeddinghaus I, Salter J, Hills M, Dixon M, Ebbs S, Gui G, Sacks N, Smith I: HER-2 amplification impedes the antiproliferative effects of hormone therapy in estrogen receptorpositive primary breast cancer. Cancer Res 2001, 61:8452-8458.

39. Oesterreich S, Zhang P, Guler RL, Sun X, Curran EM, Welshons WW, Osborne CK, Lee AV: Re-expression of estrogen receptor alpha in estrogen receptor alpha-negative MCF-7 cells restores both estrogen and insulin-like growth factor-mediated signaling and growth. Cancer Res 2001, 61:5771-5777.

40. McClelland RA, Barrow D, Madden TA, Dutkowski CM, Pamment J, Knowlden JM, Gee JM, Nicholson Rl: Enhanced epidermal growth factor receptor signaling in MCF7 breast cancer cells after long-term culture in the presence of the pure antiestrogen $\mathrm{ICl} 182,780$ (faslodex). Endocrinology 2001, 142:2776-2788.

\section{doi:10.1186/1471-2407-13-473}

Cite this article as: Milosevic et al: Clinical instability of breast cancer markers is reflected in long-term in vitro estrogen deprivation studies. BMC Cancer 2013 13:473. 